\title{
CIRUGÍA MAYOR AMBULATORIA EN UROLOGÍA. 5 AÑOS DE EXPERIENCIA
}

\author{
B. LLOPIS GUIXOT, J.A. NAVARRO ANTÓN, M.J. MOLA ARIZO, V. GONZALVO PÉREZ, \\ R. BOTELLA ALMODÓVAR, E. CANTÓ FAUBEL, A.C. POLO PERIS
}

Servicio de Urología del Hospital Lluis Alcanyís de Xàtiva. Valencia.

Actas Urol Esp. 27 (2): 117-122, 2003

\section{RESUMEN}

"CIRUGÍA MAYOR AMBULATORIA EN UROLOGÍA. 5 AÑOS DE EXPERIENCIA"

INTRODUCCIÓN: La cirugía mayor ambulatoria (CMA) comprende aquellos procedimientos quirúrgicos que requieren un escaso periodo de recuperación post-operatoria, siendo dados de alta los pacientes en el mismo día de la intervención.

OBJETIVOS: Este trabajo pretende explicar el funcionamiento de nuestro Servicio de Urología en una unidad integrada de cirugía mayor ambulatoria, así como revisar los resultados obtenidos en los cinco primeros años de vida de nuestra unidad.

MATERIAL Y MÉTODOS: Se han incluido 339 pacientes con una edad media de 37,5 años (3-84) exponiendo el protocolo de selección de pacientes, patología, procedimiento empleado y tipo de anestesia, así como los criterios de alta.

RESULTADOS: En 4 pacientes $(1,2 \%)$ ha habido complicaciones intraoperatorias en 24 (7\%) inmediatas y en $33(9,7 \%)$ tardías. Según resultados de la encuesta anónima realizada sistemáticamente a todos los pacientes el $95 \%$ se han mostrado satisfechos con esta nueva modalidad asistencial.

CONCLUSIONES: La CMA supone una mínima alteración del modo de vida del paciente, disminuye costos hospitalarios, la morbilidad es similar a la de la hospitalización y supone un reto para los profesionales del Servicio.

PALABRAS CLAVE: Cirugía Mayor Ambulatoria. Urología.

\section{ABSTRACT}

"AMBULATORY SURGERY IN UROLOGY. FIVE YEARS OF EXPERIENCE"

INTRODUCTION: The ambulatory surgery includes those surgical procedures that require a limited period of post-operative recovery, so that patients will be discharged from hospital on the same day of their surgical operation.

OBJETIVES: This publication aims at both, explaining our Service way of working as an integrated unit of ambulatory surgery, and it also tries to revise the results which have been obtained during our first 5 years working together.

METHOD AND MATERIALS: Three hundred and thirty-nine patients, with an average age of 37.5 years (3-84), have been included in this report which sets out the medical record of this patients selection, their pathologies, the different procedures used, the kind of anaesthesia as well as the criterion to discharge them from hospital.

RESULTS: Intra-surgical complications have arisen in 4 (1.2\%) of our patients, inmediate complications in 24 patients (7\%) and late ones in $33(9.7 \%)$ of our patients. According to the results of an anonymous inquiry which has been polled systematically to all our patients, $95 \%$ of those polled are completely satisfied with this new way of medical attention.

CONCLUSIONS: The ambulatory surgery means a sligth change in our patients' way of life. It also diminishes hospital costs, morbidity is similar to the one produced in hospitalization and it implies an important challenge for the professionnals of this Service.

KEY WORDS: Ambulatory Surgery. Urology. 
$\mathrm{L}^{2}$ a cirugía mayor ambulatoria (CMA) es aqueAla que realizada bajo anestesia general, regional o local, requiere un periodo variable de recuperación postoperatoria previo al alta domiciliaria, en el mismo día de la intervención. El resto de procedimientos quirúrgicos, llamados hasta ahora "ambulantes" o "locales", de baja complejidad, realizados siempre bajo anestesia local y en los que el paciente recibe un alta inmediata a su domicilio, formarán parte de la cirugía menor ambulatoria $^{1}$.

El incremento del nivel cultural y de la longevidad de la población, junto a una mayor oferta asistencial, comportan un progresivo aumento de la demanda quirúrgica, que ha puesto en evidencia la limitación de los recursos sanitarios. La CMA surge pues con la finalidad de optimizar estos recursos, mejorando además la calidad asistencial.

La gran cantidad de patología de mediana complejidad existente, la tradición y constante desarrollo de técnicas endoscópicas y la aparición de tratamientos realizados mediante efectos físicos producidos por aparatos de nueva creación (ondas de choque, láser, termoterapia, hipertermia ...) son hechos, que convierten a la Urología en una especialidad quirúrgica con gran proyección en el campo de la CMA.

\section{OBJ ETIVOS}

Este trabajo pretende explicar el funcionamiento de nuestro Servicio de Urología en una unidad integrada de cirugía mayor ambulatoria, así como evaluar los resultados obtenidos en sus primeros 5 años de existencia, haciendo hincapié en las complicaciones habidas.

\section{PACIENTES Y MÉTODOS}

La selección de pacientes es realizada de forma conjunta por el anestesista y el urólogo; está en función de la patología y se basa en unos criterios estrictos tanto "médicos" como "sociales" de inclusión y exclusión.

Los requisitos que deben reunir los procedimientos anestésico-quirúrgicos en la CMA comprenden ${ }^{1,2}$ : no precisar una preparación preoperatoria compleja; poderse realizar en un tiempo máximo de 90 minutos (si la anestesia es general) sin excesiva manipulación de tejidos y sin riesgo de pérdidas hemáticas importantes. La recuperación del paciente debe ser breve y el dolor controlable con medicación oral y no precisar medicación endovenosa; la técnica debe estar perfectamente protocolizada y ser "dominada" por el urólogo.

Las patologías contempladas en nuestro protocolo se detallan en la Tabla I. Serán condicionantes médicos de exclusión ${ }^{1-4}$ : edad menor a 3 años o peso menor a $15 \mathrm{Kg}$; riesgo quirúrgico alto (ASA III-IV), obesidad mórbida, drogadicción, tratamiento previo con IMAOS y antecedentes de hipertermia maligna.

Los condicionantes sociales incluirán ${ }^{1,3}$ : acompañante responsable, medio de transporte propio o accesible, disponer de teléfono y de vivienda que reúna unas condiciones higiénicas adecuadas, sin barreras arquitectónicas y que se encuentre situada a una distancia razonable (inferior a 45 minutos en coche) del hospital.

Tras una detallada información de su patología, tipo de intervención a realizar, alternativas al tratamiento, seguimiento postoperatorio y posibles complicaciones, el paciente o su tutor deberán aceptar el tipo de tratamiento propuesto mediante la firma del consentimiento informado ${ }^{5}$.

\section{TABLA I}

Patología inguino-escrotal:

- Varicocelectomía.

- Cirugía de la persistencia del ductus p-v.

- Funiculolisis y orquidopexia.

- Quistectomía funicular y epididimaria.

- Orquiectomía.

- Hidrocelectomía.

- Biopsia de testículo.

Patología uretro-vesical:

- Biopsia vesical.

- RTU de pequeñas neoformaciones vesicales.

- Uretrotomías cortas.

- Litotricia endoscópica.

- Cistostomía.

- Cistolitectomía.

- Carunculectomía.

Patología ureteral:

- Extracción endoscópica de cálculos.

- Meatotomía ureteral.

- Cateterismos ureterales.

- Ureterorrenoscopia.

Otras patologías:

- Nefrostomía percutánea.

- Circuncisión en niños. 
Las intervenciones se llevan a cabo a primera hora de la mañana; reciben profilaxis antibiótica sólo aquellos pacientes sometidos a procedimientos endoscópicos.

Tras la cirugía, los pacientes permanecen durante unas horas bajo los cuidados de enfermería en una sala destinada a tal fin, estando acompañados por un familiar.

Los pacientes regresan a su domicilio cuando cumplen los "criterios de alta"6 establecidos (Tabla II) recibiendo instrucciones específicas de cada grupo de patologías para su postoperatorio, donde se detalla: cuidados de la herida, analgésicos, actividad, dieta, teléfono al que recurrirán en caso de duda o complicaciones, y día y hora del próximo control en consultas externas. El paciente será ingresado en caso de no cumplir "los criterios de alta" a una hora determinada (18 horas).

\section{TABLA II}

\section{Criterios de alta}

- Consciente y orientado.

- Constantes vitales estables durante la última hora.

- Tolerancia de líquidos.

- Diuresis espontánea (excepto en algunos procedimientos urológicos).

- Deambulación autónoma sin sensación de inestabilidad.

- Ausencia de dolor intenso, sangrado de la herida, náuseas y/o vómitos.

A las 24 horas, la enfermería de la unidad contacta telefónicamente ${ }^{7}$ con el paciente para conocer su situación y a los dos días, todos los pacientes son revisados en consultas externas. Con posterioridad, en una segunda visita post-operatoria, cumplimentan un cuestionario referente al grado de aceptación del procedimiento.

\section{RESULTADOS}

Desde octubre de 1995 hasta julio del 2000 hemos intervenido en la UCMA a 339 pacientes (18\% de los pacientes intervenidos en nuestro servicio), de los cuales 10 eran mujeres y 329 varones. La edad media fue de 37,5 (3-84) años.

Hemos realizado un total de 397 procedimientos quirúrgicos que a continuación detallamos:
Cirugía inguino-escrotal:

- 77 hidrocelectomías (4 bilaterales)

- 65 varicocelectomías

- 30 quistectomías funiculares o epididimarias

-23 funiculolisis y orquidopexias

- 22 cierres de ductus peritoneo-vaginal persistente

- 12 biopsias de testículo

- 11 orquiectomías

- 6 vasectomías*

- 4 implantes de prótesis testicular

- 1 exploración quirúrgica de conducto inguinal por monorquia

- 1 exéresis de tumor de rafe medio escrotal

- 1 herniorrafia inguinal (coincidente con quiste de cordón)

- 1 Friedrich escrotal

- 1 exéresis de quistes sebáceos en escroto

Cirugia uretrovesical:

- 39 RTUs de tumor vesical

- 15 biopsias vesicales randomizadas

- 9 uretrotomías endoscópicas

- 9 cateterismos ureterales

-3 cistoscopias

- 1 extracción endoscópica de litiasis vesical

- 1 sobredistensión vesical

-1 TCP

- 1 ureteroscopia

-2 carunculectomías

Otras patologías:

- 55 circuncisiones (niños y *)

-2 frenuloplástias*

- 3 exeresis/biopsia de lesión en glande

- 1 biopsia de próstata*

*Acompañando a otros procedimientos

De los 339 pacientes, 181 fueron intervenidos con anestesia local más sedación, 46 con raquianestesia, y 122 se operaron bajo anestesia general.

Sólo los 72 pacientes sometidos a procedimientos endoscópicos recibieron profilaxis antibiótica ( 1 gramo de Cefonicida IV) 1 hora antes de la cirugía. La permanencia en la UCMA osciló entre 3 y 6 horas (estancia media: 4,2 horas). 
En nuestra serie 4 pacientes presentaron complicaciones intra-operatorias: 2 cambios de técnica quirúrgica por hallazgos incidentales (hernia inguinal coincidente con quiste de cordón, y tumor en cistoscopia); 2 alargamientos del tiempo quirúrgico (disección dificultosa del teste criptorquídico en el primero y ausencia del teste en el segundo caso).

Presentaron complicaciones inmediatas (durante su estancia en la unidad) 24 pacientes (7\%): problemas anestésicos (16), sangrado de la herida quirúrgica (4), cólico nefrítico (1), retención aguda de orina (1), excesivo dolor herida (1), hematuria (1).
Presentaron complicaciones tardías (en domicilio) 33 pacientes (9,7\%): infección de herida quirúrgica (9), orquiepididimitis post-hidrocelectomía/quistectomía de cordón (7), hematoma escroto (4), seroma herida (3), hematuria (4), infección de tracto urinario (1), dolor abdominal (1), dolor herida (1), retención aguda de orina (1), estenosis de meato (1), dolor teste (1).

Precisaron hospitalización (nunca superior a 24 horas) 26 pacientes (7,9\%): 3 por complicaciones intraoperatorias (alargamiento del tiempo quirúrgico o cambio de actitud quirúrgica), 19 por complicaciones inmediatas (Tabla III) y 4 por complicaciones tardías (Tabla IV).

\section{TABLA III}

\begin{tabular}{|c|c|c|c|c|}
\hline $\begin{array}{l}\text { Complicaciones } \\
\text { inmediatas }\end{array}$ & Cirugía & Anestesia & Edad & Hospitalización \\
\hline \multirow{4}{*}{ Sangrado herida } & Quiste de cordón & $\mathrm{L}$ & 52 & No \\
\hline & Hidrocelectomía & $\mathrm{L}$ & 53 & No \\
\hline & Varicocelectomía & $\mathrm{L}$ & 25 & No \\
\hline & Quistectomía de cordón & $\mathrm{L}$ & 67 & $\mathrm{Si}$ \\
\hline \multirow{16}{*}{ Problemas anestésicos } & Biopsia vesical & $\mathrm{R}$ & 72 & $\mathrm{Si}$ \\
\hline & Orquidopexia+cierre ductus & $\mathrm{G}$ & 7 & $\mathrm{Si}$ \\
\hline & Cierre ductus & $\mathrm{G}$ & 7 & $\mathrm{Si}$ \\
\hline & Cierre ductus & $\mathrm{G}$ & 9 & $\mathrm{Si}$ \\
\hline & Carunculectomía & $\mathrm{L}$ & 55 & $\mathrm{Si}$ \\
\hline & Varicocelectomía & $\mathrm{L}$ & 26 & $\mathrm{Si}$ \\
\hline & Prótesis teste & $\mathrm{L}$ & 18 & $\mathrm{Si}$ \\
\hline & Funiculolisis+orquidopexia & $\mathrm{G}$ & 11 & $\mathrm{Si}$ \\
\hline & Varicocelectomía & $\mathrm{L}$ & 26 & $\mathrm{Si}$ \\
\hline & Circuncisión+prótesis teste & $\mathrm{L}$ & 18 & $\mathrm{Si}$ \\
\hline & Cierre ductus & G & 5 & $\mathrm{Si}$ \\
\hline & Hidrocelectomía & $\mathrm{L}$ & 20 & $\mathrm{Si}$ \\
\hline & Circuncisión & G & 5 & $\mathrm{Si}$ \\
\hline & Hidrocelectomía & $\mathrm{G}$ & 21 & $\mathrm{Si}$ \\
\hline & Hidrocelectomía & $\mathrm{L}$ & 75 & si \\
\hline & RTU Tm vesical & $\mathrm{R}$ & 70 & $\mathrm{Si}$ \\
\hline Cólico nefrítico & Cateterismo ureteral & $\mathrm{R}$ & 47 & $\mathrm{Si}$ \\
\hline R.A.O. & Orquiectomía & $\mathrm{R}$ & 63 & No \\
\hline Excesivo dolor herida & Varicocelectomía & $\mathrm{L}$ & 19 & No \\
\hline Hematuria & Biopsia vesical randomizada & $\mathrm{R}$ & 75 & $\mathrm{Si}$ \\
\hline
\end{tabular}


TABLA IV

\begin{tabular}{|c|c|c|c|c|}
\hline $\begin{array}{l}\text { Complicaciones } \\
\text { tardías }\end{array}$ & Cirugía & Anestesia & Edad & Ingreso \\
\hline \multirow{9}{*}{ Infección herida/Abceso escrotal } & Hidrocelectomía & $\mathrm{L}$ & 50 & No \\
\hline & Hidrocelectomía & $\mathrm{L}$ & 52 & No \\
\hline & Hidrocelectomía & $\mathrm{L}$ & 16 & No \\
\hline & Hidrocelectomía & $\mathrm{L}$ & 49 & $\mathrm{Si}$ \\
\hline & Quistectomía cordón & $\mathrm{L}$ & 73 & No \\
\hline & Varicocelectomía & $\mathrm{L}$ & 26 & No \\
\hline & Cirugia criptorquidia & $\mathrm{G}$ & 10 & No \\
\hline & Hidrocelectomía & $\mathrm{G}$ & 56 & No \\
\hline & Quistectomía cordón & $\mathrm{L}$ & 63 & No \\
\hline \multirow{3}{*}{ Seroma herida } & Varicocelectomía & $\mathrm{G}$ & 21 & No \\
\hline & Hidrocelectomía & $\mathrm{L}$ & 50 & No \\
\hline & Varicocelectomía & $\mathrm{L}$ & 14 & No \\
\hline \multirow{7}{*}{ Orquiepididimitis } & Hidrocelectomía & $\mathrm{L}$ & 16 & No \\
\hline & Quistectomía epidid. & $\mathrm{L}$ & 63 & No \\
\hline & Hidrocelectomía & $\mathrm{L}$ & 68 & No \\
\hline & Varicocelectomía & $\mathrm{L}$ & 31 & No \\
\hline & Hidrocelectomía & $\mathrm{L}$ & 19 & No \\
\hline & Quistectomía cordón & $\mathrm{L}$ & 63 & No \\
\hline & Hidrocelectomia & $\mathrm{L}$ & 71 & No \\
\hline \multirow{4}{*}{ Hematoma escroto } & Hidrocelectomía & $\mathrm{L}$ & 61 & No \\
\hline & Quistectomía cordón & $\mathrm{L}$ & 61 & No \\
\hline & Hidrocelectomía & $\mathrm{L}$ & 19 & No \\
\hline & Hidrocelectomía & $\mathrm{L}$ & 18 & No \\
\hline \multirow{4}{*}{ Hematuria } & RTU Tm vesical & $\mathrm{R}$ & 60 & $\mathrm{Si}$ \\
\hline & Cistoscopia+cateterismo ureteral & $\mathrm{R}$ & 47 & $\mathrm{Si}$ \\
\hline & Pielografía retróg. + Biop. próstata & $\mathrm{G}$ & 75 & No \\
\hline & Biopsia vesical aleatoria & $\mathrm{R}$ & 73 & No \\
\hline Infección de orina & Uretrotomia interna & $\mathrm{G}$ & 52 & No \\
\hline
\end{tabular}

Según los resultados de la encuesta anónima realizada a los pacientes a los 15-20 días del alta, el 95\% de los pacientes se muestran "satisfechos" o "muy satisfechos" de haber sido operados sin hospitalización y repetirían la experiencia en caso necesario. Sólo el 5\% se mostraron "poco o nada satisfechos" con el carácter ambulatorio de su cirugía.

\section{DISCUSION - CONCLUSIONES}

En octubre de 1995, nuestro servicio comenzó a trabajar en la unidad integrada de cirugía mayor ambulatoria de nuestro hospital, con la incertidumbre que suponía este nuevo modelo asistencial, aunque animados por los buenos resultados conocidos por la literatura ${ }^{8-10}$ y la ilusión de los profesionales del Servicio. 
Nuestra experiencia ha puesto de manifiesto, como bien dice Davis, ${ }^{11}$ que la CMA es un fenómeno "raro" en el cual todos los participantes se benefician.

La CMA comporta una reducción de los costos sanitarios, la mayor parte de los cuales dependen del ahorro en hostelería, tal y como ha demostrado el sistema informático-económico de nuestro centro. Del mismo modo aumenta el número de camas disponibles.

El paciente, como revela nuestra encuesta, se muestra "satisfecho" o "muy satisfecho" con esta nueva modalidad asistencial (95\%), pues altera mínimamente su modo de vida, al volver a su domicilio a las pocas horas de ser operado. De manera especial se muestran agradecidos los padres de los pacientes pediátricos. La ansiedad del enfermo se ve reducida tanto por no verse acompañados de enfermos más graves, como por el hecho de asociar la rápida vuelta a casa, con una intervención de no demasiada magnitud.

Para el cirujano, la CMA, supone una mayor motivación profesional a la vez que un mayor índice de satisfacción a la vista de los buenos resultados obtenidos.

Hemos tenido una baja tasa de complicaciones (18\%), todas ellas menores ${ }^{12}$, en ningún caso superior a la de los pacientes hospitalizados. Los problemas anestésicos suponen el $67 \%$ de las complicaciones inmediatas, siendo todos ellos causa de ingreso. La práctica totalidad de las complicaciones tardias han sido de origen quirúrgico destacando entre ellas las infecciones/hematomas derivados de la cirugía escrotal (56\%).

Sólo el 8\% de los pacientes han precisado hospitalización (nunca superior a 24 horas), no requiriendo ninguno de ellos ser reintervenido.

Hoy la CMA se ha convertido en una parte importante (18\%) de la actividad quirúrgica de nuestro Servicio y a medida que ganamos en experiencia vamos "relajando" los criterios de selección e incluyendo procedimientos de mayor complejidad.

\section{REFERENCIAS}

1. Comissió per l'elaboració y remenacions pel desenvolupament de la cirugía ambularoria. Academia de ciencies médiques de Catalunya i Balears. Societat catalana de cirugía. Coordinador: Jordi Colomer. Barcelona maig-1993.

2. HOLLANDER LF, MEYER C, MANZINI N.: Critères de selection et contre-indications de la chirugie ambulatoire. Cirugie 1990; 116: 568-572.

3. DAVIS JE, SUGIOKA K.: Selección de pacientes para cirugía mayor ambulatoria: evaluación quirúrgica y anestésica. Clin Quir Nort 1987; 4: 737-748.

4. MERIDY HW.: Criteria for selection of ambulatory sugical patiens and guid lines for anethesia management. A retrospective study of 1553 cases. Ansth Anals 1982; 61: 921-926.

5. VICENTE FUENTES F.: Aspectos legales. En: Porrero J.L. Cirugía Mayor Ambulatoria. Manual práctico. Ed. Doyma S.A. 1999: 389-396.

6. MORO BLÁZQUEZ B, DOMINGO TRIALDÓ V.: Criterios de alta en cirugía ambulatoria. En: Carrasco Jiménez M.S. Anestesia para la cirugía ambulatoria. Tomo II. Barcelona: Edika-Med 1999: 219-238.

7. RIESTRA GÓMEZ A, BUSTAMANTE RUIZ A.: Control post-operatorio de los pacientes intervenidos en una unidad de Cirugia Mayor Ambulatoria. En: Maestre Alonso J.M. Guía para la planificación y desarrollo de un programa de Cirugía Mayor Ambulatoria. Ed. Ergon 1997: 203-206.

8. VARGAS C, RIUS G.: Cirugía urológica sin ingreso. Actas Urol Esp 1993; 17: 87.

9. SAMPIETRO A, VAQUERIZO A y cols: Cirugía Mayor Ambulatoria en Urología: nuestra experiencia. Arch Esp Urol 1995; 48 (4): 343-346.

10. CALDAMONE A, RABINOWITZ R.: Outpatient orchiopexy. J Urol 1982; 127: 286.

11. DAVIS JE.: Futuro de la Cirugía Mayor Ambulatoria. Clin Quir Nort 1987; 4: 921-930.

12. NATOF HE.: Complications. En: Wetchler B.V., ed. Anaesthesia for ambulatory surgery. Filadelfia: J.B. Lippincott C.O., 1990: 321-356.

Dr. B. Llopis Guixot

Servicio de Urología

Hospital Lluis Alcanyís

Ctra. Xátiva-Silla, km. 2

46800 Xátiva (Valencia)

(Trabajo recibido el 17 diciembre de 2001) 